AIAA-94-0086

N95- 13720

07307

322762

\title{
Measured and Calculated Spectral \\ Radiation from a Blunt Body Shock Layer in an Arc-Jet Wind Tunnel
}

Roger Craig MCAT Institute

Moffett Field

California 
-

$\overline{\underline{\underline{i}}}$
$\underline{\underline{\underline{\underline{z}}}}$

竞 


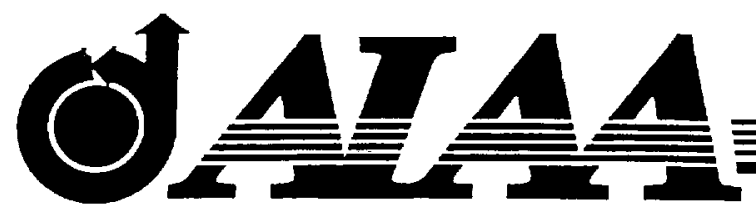

\section{AIAA 94-0086}

Measured and Calculated Spectral Radiation from a Blunt Body Shock Layer in an Arc-Jet Wind Tunnel

Dikran S. Babikian and Giuseppe Palumbo Eloret Institute Palo Alto, CA 94303

Roger A. Craig MCAT Institute Moffett Field, CA 94035 and

Chul Park, Grant Palmer, and Surendra P. Sharma NASA Ames Research Center Moffett Field, CA 94035

\section{AIAA 32nd Aerospace Sciences Meeting} January 10-13,1994 / Reno, Nevada 


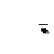

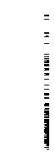

咅 


\title{
MEASURED AND CALCULATED SPECTRAL RADIATION FROM A BLUNT BODY SHOCK LAYER IN AN ARC-JET WIND TUNNEL
}

\author{
Dikran S. Babikian* and Giuseppe Palumbo* \\ Eloret Institute, Palo Alto, CA 94303 \\ Roger A. Craig** \\ MCAT Institute, Moffett Field, CA 94035 \\ and \\ Chul Park ${ }^{\dagger}$, Grant Palmer ${ }^{*}$ and Surendra P. Sharma $\ddagger$ \\ NASA Ames Research Center, Moffett Field, CA 94035
}

\begin{abstract}
Spectra of the shock layer radiation incident on the stagnation point of a blunt body placed in an arc-jet wind tunnel were measured over the wavelength range from 600 $\mathrm{nm}$ to $880 \mathrm{~nm}$. The test gas was a mixture of $80 \%$ air and $20 \%$ argon by mass, and the run was made in a highly nonequilibrium environment. The observed spectra contained contributions from atomic lines of nitrogen, oxygen, and argon, of bound-free and free-free continua, and band systems of $\mathrm{N}_{2}$ and $\mathrm{N}_{2}^{+}$. The measured spectra were compared with the synthetic spectra, which were obtained through four steps: the calculation of the arc-heater characteristics, of the nozzle flow, of the blunt-body flow, and the nonequilibrium radiation processes. The results show that the atomic lines are predicted approximately correctly, but all other sources are underpredicted by orders of magnitude. A possible explanation for the discrepancy is presented.
\end{abstract}

\section{Nomenclature}

A = Einstein A-coefficient.

E = Energy level of emitting state, $\mathrm{cm} .^{-1}$

8 = Statistical weight of the emitting state.

b $\nu=$ Photon energy, $\mathrm{cm}^{-1}$.

$I$ = Intensity of a spectral line, $\mathrm{W} /\left(\mathrm{cm}^{2}-\mu-\mathrm{sr}\right)$.

$T$ = Heavy particle translational temperature, $K$.

$T_{e}=$ Electron-electronic temperature, $K$.

$T_{v}=$ Vibrational temperatue, $\mathrm{K}$.

$\mathrm{V}=$ Flight velocity, $\mathrm{km} / \mathrm{s}$.

\section{Introduction}

For the last few decades, characterization of the radiation phenomena in the nonequilibrium region of a shock layer over a blunt body flying in high velocity, low density environments has been the focus of the efforts of many researchers. During an atmospheric entry, a spacecraft pases through the regime of altitudes where the flow be-

This paper is declared a work of the U.S. Government, and is not subject to copyright protection in the United States.

- Research Scientist; Member AIAA.

- Research Scientist; Senior Member AIAA.

†Staff Scientist; Associate Fellow AIAA.

$\ddagger_{\text {Research Scientist; Associate Fellow AIAA. }}$ hind the shock wave is in nonequilibrium in both chemical composition and internal thermal modes. It is known that the intensity of the radiation is strongest in the middle of the nonequilibrium zone. ${ }^{1}$ For entry speeds in excess of 10 $\mathrm{km} / \mathrm{s}$ for Earth and $8 \mathrm{~km} / \mathrm{s}$ for Mars and Venus, nonequilibrium radiation is the major source of heat transfer to the stagnation region. ${ }^{1,2}$ For this reason, a flight experiment, called the Aeroassist Flight Experiment (AFE), had been proposed at one time for the purpose of determining the intensity of this radiation. 3,4

Predicting the flowfield around an entry body for an equilibrium regime and the radiation emitted by the equilibrium fowfield can be done more confidently; predicting nonequilibrium radiation is difficult because of the complex nature of the nonequilibrium radiation phenomena. However, in the last few years, through concerted theoretical $^{5-8}$ and experimental ${ }^{9-12}$ efforts, it became possible to predict the radiation features in the region of peak nonequilibrium radiation with fair accuracy. This is accomplished by using a computer code named Nonequilibrium (NONEQ), ${ }^{8}$ which is an expanded version of the code Nonequilibrium Air Radiation program (NEQAIR), ${ }^{6}$ which accounts for the nonBoltzmann populations of electronic states and carries out detailed line-by-line calculation of both atomic and molecular lines.

Questions remain as to how well the NONEQ code predicts the radiation phenomena in the very low density regime where the nonequilibrium peak does not occur in the shock layer. That is, the truncated flow where the available flow time is shorter than the time needed for the flow to reach the peak nonequilibrium radiation point. Recently, two flight experiments, named Bow Shock I and Bow Shock 2, have been conducted in which the NO Gamma band radiation incident on the stagnation point was measured at flight speeds of 3.5 and $5.2 \mathrm{~km} / \mathrm{s}$, respectively. ${ }^{13}$ The results show that the NONEQ code predicts the radiation intensities quite well in the equilibrium and moderately nonequilibrium regimes but underestimates it by many orders of magnitude in severely nonequilibrium regimes. The discrepancy between the theory and measurement is less for the $5.2 \mathrm{~km} / \mathrm{s}$ flight than for the $3.5 \mathrm{~km} / \mathrm{s}$ flight. Despite intensive theoretical study of this phenomenon, the discrepancy has not yet been satisfactorily explained. ${ }^{13}$

The flow regime of this large discrepancy is illustrated in Fig. 1. The figure shows the behavior of the trans- 
lational temperature $T$, vibrational temperature $T_{v}$, and radiation emission power behind a normal shock wave in a one-dimensional flow computed using the NONEQ code. The freestream conditions are those of the present experiment to be described in detail later. As seen here, the radiation emission peaks. If the shock layer thickness is larger than the distance to the peak radiation point, as indicated in the figure as the AFE vehicle condition, then the flowfield can be said to be in moderate nonequilibrium. The existing NONEQ and similar codes correctly predict the total radiation power emitted in this case. A highly nonequilibrium flowfield is one in which the shock layer thickness is shorter than the distance to the peak radiation point, as indicated in the figure as the present arc-jet test condition. The discrepancy between the measurements and calculations for the Bow Shock flights occurred when the flow environment was in this highly nonequilibrium regime.

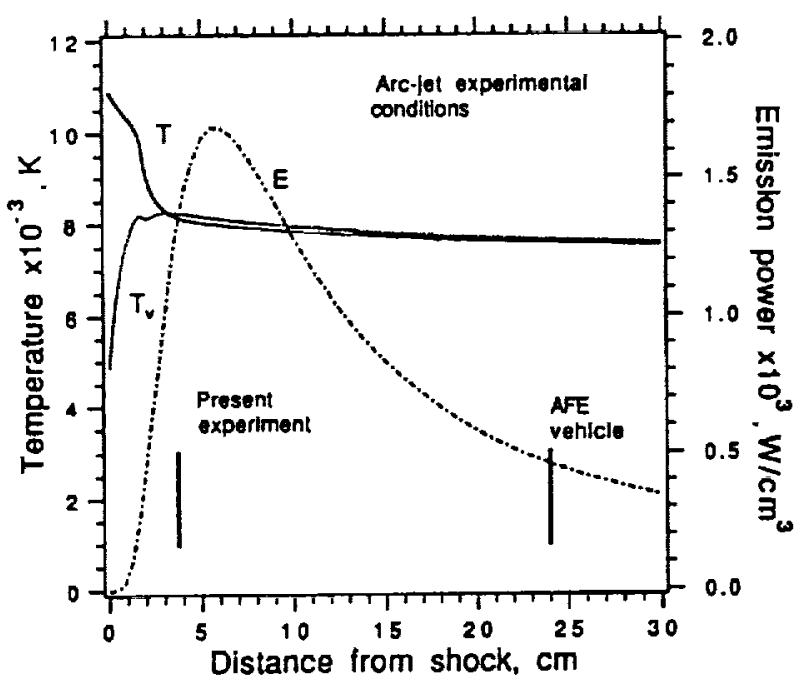

Fig. 1. Comparison between the relaxation distance and flow lengths for the present experimental condition.

The purpose of the present work is to study the radiation phenomena in this highly nonequilibrium regime, where, as shown in Fig. 1, the available flow distance is shorter than the distance needed for the flow to reach peak radiation. The study is conducted at an enthalpy corresponding to a flight speed of $7.8 \mathrm{~km} / \mathrm{s}$. If the trend observed in the flight experiment holds true, that the discrepancy is smaller at higher flight velocity, one would hope that there would be little or no discrepancy between the present data and the calculations made using the NONEQ code at this enthalpy level. If the discrepancy between experiment and calculation still exists, the study should contribute to elucidate the nature of the discrepancy.

The experimental work is carried out with a model placed in the test section of an arc-heated wind tunnel. The radiation incident on the stagnation point of a flat circular disk is detected using a grating spectrograph.
Observations were made in the near infrared wavelength range from 600 to $880 \mathrm{~nm}$. This spectral region is chosen because it contains strong and well characterized lines from nitrogen and oxygen atoms and molecular band radiation from the $\mathrm{N}_{2}$ First Positive system. In addition, there are bound-free and free-free continua that can potentially contribute to the overall background radiation. By comparing the intensities of these different radiation sources, an insight about the nature of the highly nonequilibrium regime can be gained.

In order to produce a synthetic spectrum for the experimental condition, the thermochemical state of the flow in the test section of the arc-jet wind tunnel is calculated using the best available computer code. ${ }^{14}$ The enthalpy value input into the code is calculated from the arc heater characteristics ${ }^{15}$ and the results of the heat transfer measurements to a sphere placed in the freestream. The nonequilibrium relaxation phenomena in the shock layer are then calculated using both the one-dimensional NONEQ code and a two-dimensional code. ${ }^{16}$ Little difference was seen between the results of the one- and the twodimensional calculations. The radiative transport calculation was then carried out through the shock layer using the NONEQ code.

When the results of the computations are compared with the experimental data, it is found that the computations severely underestimate the intensity of continua and molecular bands by several orders of magnitude. Thus, it is concluded that the trend seen in the flight experiments at 3.5 and $5.2 \mathrm{~km} / \mathrm{s}$ still prevails at the equivalent flight speed of $7.8 \mathrm{~km} / \mathrm{s}$. No satisfactory explanation for this discrepancy has been found.

\section{Experiment}

\section{Experimental Setup}

The experimental procedures are presented in detail in Refs. 17 and 18. However, a brief description is given here for completeness. Fig. 2 shows the schematic of the overall arrangement of the model and the radiation detection system. The experiment is conducted in a constricted arc wind tunnel at Ames Research Center rated at $20 \mathrm{MW}$, known as the Aerodynamic Heating Facility. The test was made at one run condition, which is given in Table 1 . The area ratio of the nozzle, deduced from Pitot impact pressure measurements at the station where the model was located, is 113 .

Table 1. The operating conditions of the arc-heater.
Electrical current

Electrical voltage

Electrical power input

Test gas mass flow rate

Test gas mixture by mass

Arc-heater efficiency

Mass-averaged enthalpy

Centerline enthalpy
950 amperes

1800 volts

$1.71 \mathrm{MW}$

$0.034 \mathrm{~kg} / \mathrm{s}$

$80 \%$ air, $20 \%$ Ar

$-0.43$

$21.5 \mathrm{MJ} / \mathrm{kg}$

$57.3 \mathrm{MJ} / \mathrm{kg}$ 
In addition to air and argon, a small amount of copper is in the stream, which, based on the mass loss of the electrodes, is judged to be about $50 \mathrm{ppm}$ by volume on the average. ${ }^{14}$ Vaporization of copper electrodes occurs mostly during the start-up and shut-down processes. During the run, copper vaporization is small when the settling chamber pressure is low. At 1 atm settling chamber pressure maintained in the present study, the copper content in the flow is believed to be much less than $50 \mathrm{ppm}$. Such low concentration of copper vapor is inconsequential to the flow properties, ${ }^{14}$ and therefore the presence of copper is neglected.

In order to determine the flow enthalpy, the characteristics of the arc discharge in the arc-constrictor are first calculated using the code Arc Heater Flowfield (ARCFLO). ${ }^{15}$ The code yielded the centerline enthalpy value of $57 \mathrm{MJ} / \mathrm{kg}$ at the exit of the constrictor. In the past, the centerline enthalpy values have been compared with the enthalpy values deduced from the heat transfer rates to the stagnation point of a sphere placed in the flow. The heat transfer rate-deduced enthalpy values were slightly more than half the centerline enthalpy value at the exit of the arc constrictor. The difference is believed to be caused by the mixing in the settling chamber placed between the exit plane of the arc constrictor and the nozzle entrance. ${ }^{20}$ Based on this observation, the enthalpy in the test section is judged to be approximately $30 \mathrm{MJ} / \mathrm{kg}$. The equilibrium conditions based on this enthalpy value and the known pressure in the settling chamber are given in Table 2.

Table 2. Calculated settling chamber conditions.

\begin{tabular}{ll}
\hline Pressure & $1.02 \mathrm{~atm}$ \\
Temperature & $7780 \mathrm{~K}$ \\
Centerline enthalpy & $30 \pm 3 \mathrm{MJ} / \mathrm{kg}$ \\
$\mathrm{Ar}$ mole fraction & $8.88^{-2}$ \\
$\mathrm{~N}_{2}$ mole fraction & $7.29^{-2}$ \\
$\mathrm{O}_{2}$ mole fraction & 0 \\
$\mathrm{NO}$ mole fraction & $9.20^{-4}$ \\
$\mathrm{~N}$ mole fraction & $6.29^{-1}$ \\
$\mathrm{O}$ mole fraction & $2.06^{-1}$ \\
$\mathrm{Ar}^{+}$mole fraction & $8.44^{-5}$ \\
$\mathrm{~N}^{+}$mole fraction & $7.23^{-4}$ \\
$\mathrm{O}^{+}$mole fraction & $1.94^{-4}$ \\
$\mathrm{NO}$ & $2.22^{-4}$ \\
$\mathrm{e}^{-}$mole fraction & $1.22^{-3}$ \\
\hline
\end{tabular}

Fig. 3 shows a schematic view of the model. The model face is a flat circular disk $15 \mathrm{~cm}$ in diameter with the edge rounded to approximately $3 \mathrm{~mm}$ radius. A small aperture centered on the forward face admits the surface radiative flux. The viewing optical axis is canted $15^{\circ}$ from the centerline in order to reject the direct irradiation from the arc constrictor. A magnesium-fluoride window, cooled with a flow of helium, is placed in a cavity below the aperture to transmit the radiation signal into the optical system. The optical system consists of two flat mirrors and two concave mirrors. The first flat mirror turns the direction of the oncoming beam to the first concave mirror (see Fig. 2). The parallel beam produced by this concave mirror is refocused by the second concave mirror. The beam from the second concave mirror is turned by the second flat mirror and directed into the entrance slit of a grating spectrograph. The system is water-cooled, and the optical path was evacuated during the test in order to eliminate the uncertainty caused by absorption.

During the run, a luminous shock layer is visible to the eye. The stand-off distance of the visible shock layer was determined to be approximately $4 \mathrm{~cm}$.

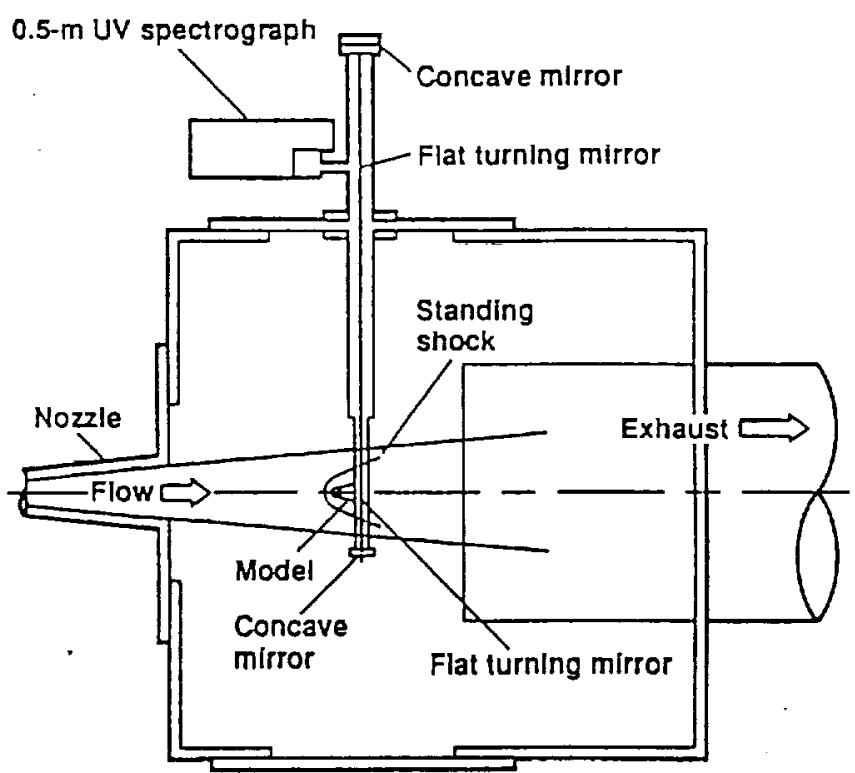

Fig. 2. Schematic of the present experimental setup in the Ames $20 \mathrm{MW}$ Aerodynamic Facility.

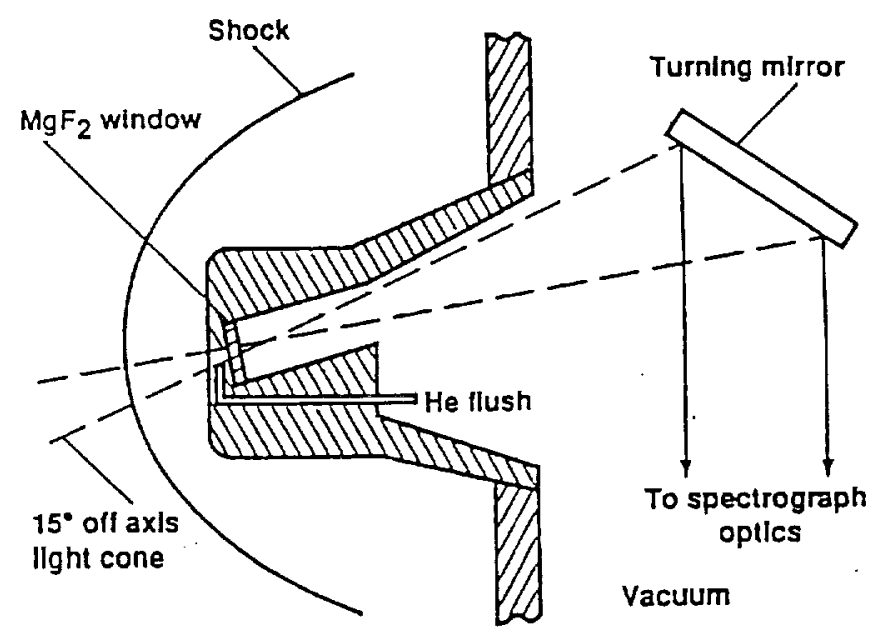

Fig. 3. Schematic of the optical arrangement within the model. 
The spectrograph was a McPherson model 216.5 Czerney-Turner instrument with a focal length of $0.5 \mathrm{me}-$ ter with an exit film plane of a $10 \mathrm{~cm}$ span. With a grating of 300 grooves per millimeter, the instrument has a reciprocal dispersion of $6.6 \mathrm{~nm} / \mathrm{mm}$ and a theoretical resolution of $0.13 \mathrm{~nm}$, and allows a spectral range of 660 $\mathrm{nm}$ to be recorded in a single exposure. As mentioned in Introduction, the radiation measurement was made for the near infrared wavelength range from 600 to $900 \mathrm{~nm}$. This region contains a number of spectral lines from nitrogen and oxygen atoms with known transition probabilities and the well-characterized $\mathrm{N}_{2}$ First Positive band system. The sensitivity of the film used declines rapidly beyond $880 \mathrm{~nm}$. In separate measurements, radiation from the visible range from 300 to $600 \mathrm{~nm}$, and that from the vacuum ultraviolet range from 120 to $300 \mathrm{~nm}$, were also made. Preliminary results for the visible and the vacuum ultraviolet spectra are presented in Refs. 18 and 19 , respectively. In addition, spectral measurements were made in the visible wavelength range from the direction normal to the nozzle axis to obtain the radiation intensities from the shock layer as a function of the distance from the model wall. This work will be presented in the future also. The present measurements were made photographically because the radiation signal was too weak to be measured reliably with a photoelectric method while maintaining a sufficiently high wavelength resolution.

A grating with 300 grooves $/ \mathrm{mm}$ blazed at $500 \mathrm{~nm}$ was used for the experiment. In the experiment, an exposure of 8 min was made on a Kodak High Speed Infrared Film. Exposures were also made of a calibrated tungsten ribbon lamp on the same film, all for the same $8 \mathrm{~min}$ exposure.

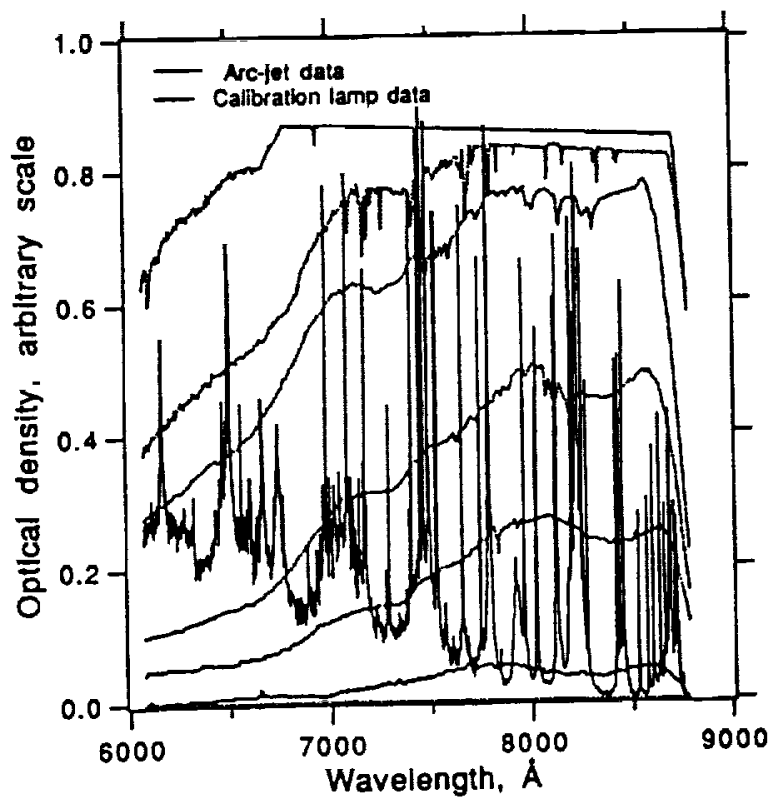

Fig. 4. Microphotodensitometer traces of the spectra obtained in the arc-jet test and the calibration lamp with varying slit widths.
The lamp was placed in front of the model, and the luminous surface of its heated tungsten ribbon was imaged on the stagnation point using a concave mirror. The solid angle of this setup was identical to that of the arc-jet experiment. The exposure from the calibration lamp was controlled by the combination of a neutral density filter and the width of the entrance slit. Second order effect was negligible for the calibration lamp.

\section{Photographic Data}

The microphotodensitometer trace of the photographic data of the arc-jet test is compared with those of the calibration lamp in Fig. 4. The ordinates in this figure represent optical densities of the film, that is, the logarithm of the attenuation of a light beam through the film. As seen here, the spectral intensities in the arc-jet data are approximately within the range covered by the calibration traces. The absolute intensity values of the arc-jet data are determined at each wavelength through interpolation between the calibration values. This was done at wavelength intervals of $0.017 \mathrm{~nm}$, resulting in a total of 16862 wavelength points. At each wavelength point, an intensity-optical density curve is constructed as shown in Fig. 5. The calibration points were fitted with a Gaussian curve using a least-square curve-fit method. The radiation intensity is deduced from the optical density of the experimental data using this curve.

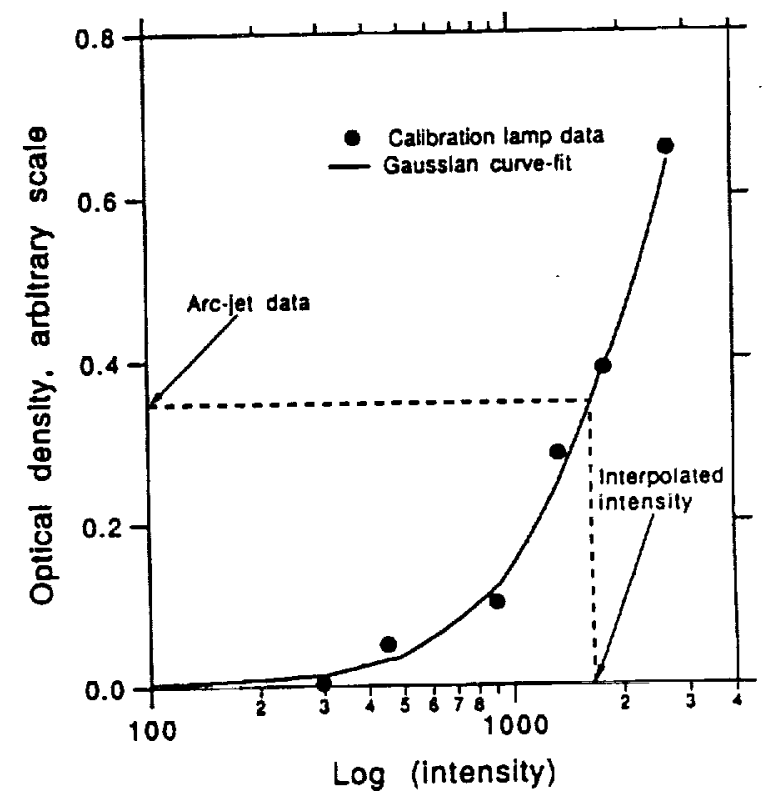

Fig. 5. The relationship between optical density and radiation intensity.

The spectrum contains the distinct features of atomic lines and complicated background features. Most of the atomic lines are identified to belong to either nitrogen, oxygen, argon, or copper. There are still unidentified lines, however. Identifiable features of the $\mathrm{N}_{2}$ First Positive band system were seen throughout the observed wave- 
length regions, and a weak feature of the $\mathrm{N}_{2}^{+}$First Negative system was identified at wavelengths below $670 \mathrm{~nm}$. Second-order images were too weak to be identified.

The area under the curve for each atomic line was calculated in order to determine the overall average line intensity over the inhomogeneous shock layer. These intensity values are plotted in an atomic Boltzmann plot in Fig. 6. As seen in the figure, the three atomic oxygen lines give an equivalent average Boltzmann temperature of about $27000 \mathrm{~K}$, whereas the nitrogen lines give about $12000 \mathrm{~K}$. These equivalent temperatures are considerably higher than the equilibrium temperatures listed in Table 2. This is a consequence of strong nonequilibrium phenomenon in the shock layer.

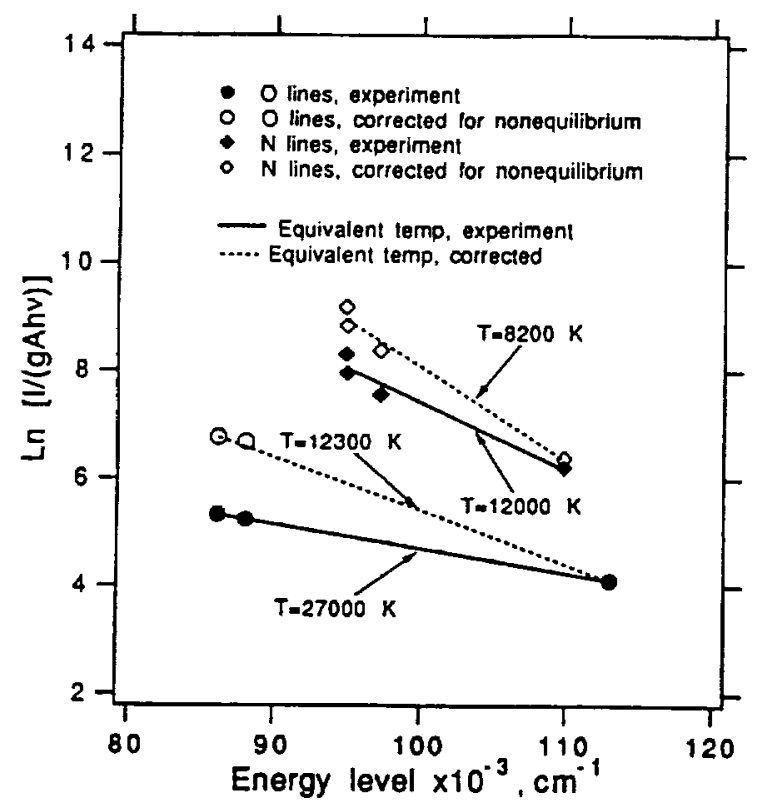

Fig. 6. Atomic Boltzmann plot of the selected atomic oxygen and nitrogen lines obtained from the experiment.

It is known that the lower excited states tend to be underpopulated with respect to the equilibrium values at low densities due to radiative depopulation. This is because (i) the radiative depopulation rate (Einstein A-coefficient) is larger for lower states than for the upper states, and (ii) the upper states tend to be in equilibrium with the free state. The extent of this underpopulation can be calculated using the NONEQ code. The calculation shows that the emitting levels of the oxygen lines in Fig. 6 emanating at energy levels around $87000 \mathrm{~cm}^{-1}$ are underpopulated by a factor of 0.23 with respect to the equilibrium value, while the population of the emitting levels around 113000 $\mathrm{cm}^{-1}$ is unaffected. For nitrogen, the lower and the upper groups of the levels are depopulated by factors of 0.41 and 0.86 , respectively. When this phenomenon is accounted for, the deduced effective average temperatures over the shock layer are $12300 \mathrm{~K}$ for oxygen and $8200 \mathrm{~K}$ for nitrogen, as shown in the figure. These values are closer to thie equilibrium temperature given in Table 2.

\section{Comparison With Calculations}

\section{Computational Method}

The thermochemical nonequilibrium relaxation processes in the nozzle are calculated using the one-dimensional Nonequilibrium $n$-Temperature (NOZNT) code. ${ }^{14}$ The code recognizes the differences among the vibrational temperatures of different molecules but assumes the electronic excitation to be characterized by the electron temperature. The electron-electronic temperature is not equated to the vibrational temperature of any species. The code has been validated against the existing experimental data in Refs. 14 and 21.

Table 3. Test section freestream conditions (NOZNT solution).

\begin{tabular}{ll} 
Geometrical area ratio of nozzle & 113 \\
Centerline enthalpy & $30 \pm 3 \mathrm{MJ} / \mathrm{kg}$ \\
Equivalent fight speed & $7.75 \mathrm{~km} / \mathrm{s}$ \\
Static pressure & $9.6^{-5} \mathrm{~atm}$ \\
Density & $5.60^{-5} \mathrm{~kg} / \mathrm{m}^{3}$ \\
Heavy particle temperature & $321 \mathrm{~K}$ \\
$\mathrm{~N}_{2}$ vibrational temperature & $4100 \mathrm{~K}$ \\
NO vibrational temperature & $910 \mathrm{~K}$ \\
Electron-electronic temperature & $6170 \mathrm{~K}$ \\
Mean free path & $0.123 \mathrm{~cm}$ \\
Equivalent flight altitude & $71.6 \mathrm{~km}$ \\
Velocity & $4890 \mathrm{~m} / \mathrm{s}$ \\
Mach number & 10.1 \\
Total number density & $2.13^{15} \mathrm{~cm}-3$ \\
Ar mole fraction & $9.30^{-2}$ \\
$\mathrm{~N}_{2}$ mole fraction & $1.22^{-1}$ \\
$\mathrm{O}_{2}$ mole fraction & 0 \\
NO mole fraction & $2.55^{-3}$ \\
$\mathrm{~N}$ mole fraction & $5.66^{-1}$ \\
O mole fraction & $2.15^{-1}$ \\
Ar mole fraction & $6.88^{-5}$ \\
$\mathrm{~N}^{+}$mole fraction & $4.93^{-4}$ \\
$\mathrm{O}^{+}$mole fraction & $1.53^{-4}$ \\
NO ${ }^{+}$mole fraction & $1.33^{-17}$ \\
$\mathrm{e}^{-}$mole fraction & $7.15^{-4}$ \\
Model & \\
Shape & flat circular disk \\
Diameter & $15 \mathrm{~cm}$ \\
Shock standoff distance & $\approx 4 \mathrm{~cm}$ \\
Equivalent sphere radius in flight & $\approx 60 \mathrm{~cm}$ \\
Knudsen number (for $15 \mathrm{~cm}$ ) & 0.0328 \\
Reynolds number (for $15 \mathrm{~cm}$ ) & 2700 \\
\hline
\end{tabular}

In Figs. 7(a) and (b), the calculated temperatures and species concentrations along the nozzle axis are shown for the present test conditions. Selected numerical values are presented in Table 3 for the nozzle exit conditions. As seen here, the vibrational temperatures of $\mathrm{N}_{2}$ and $\mathrm{NO}$ are considerably different, with $\mathrm{N}_{2}$ having the higher vibrational temperature. The electron-electronic temperature 
is considerably higher than the $\mathrm{N}_{2}$ vibrational temperature. The flow is virtually frozen in the nozzle. Most of the vibrational energy is contained in $\mathrm{N}_{2}$. The vibrational energy of $\mathrm{N}_{2}$ is larger than the electron-electronic energy at the exit of the nozzle.

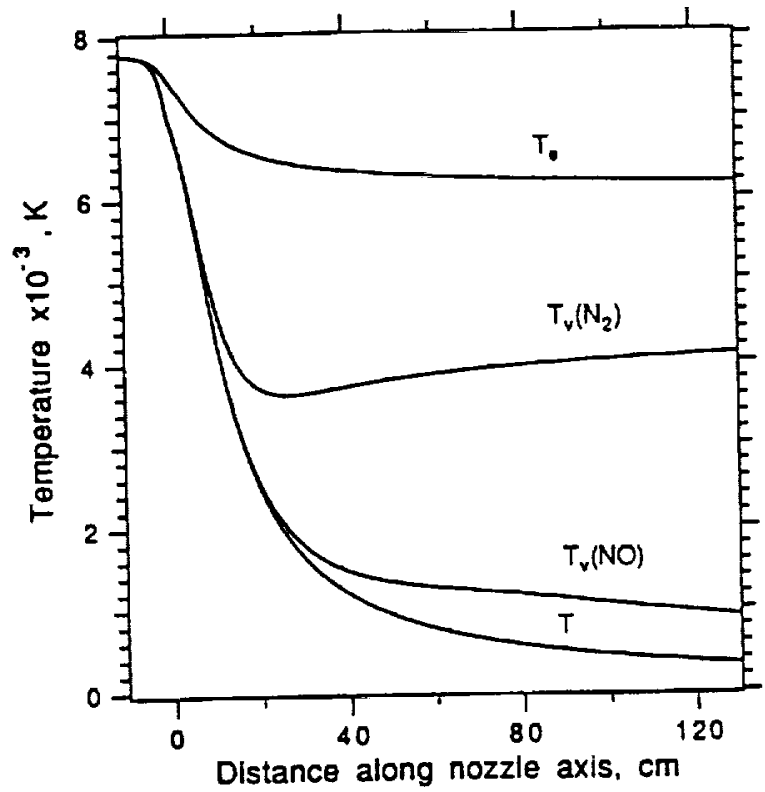

Fig. 7. Calculated nozzle flow properties. (a) Temperatures

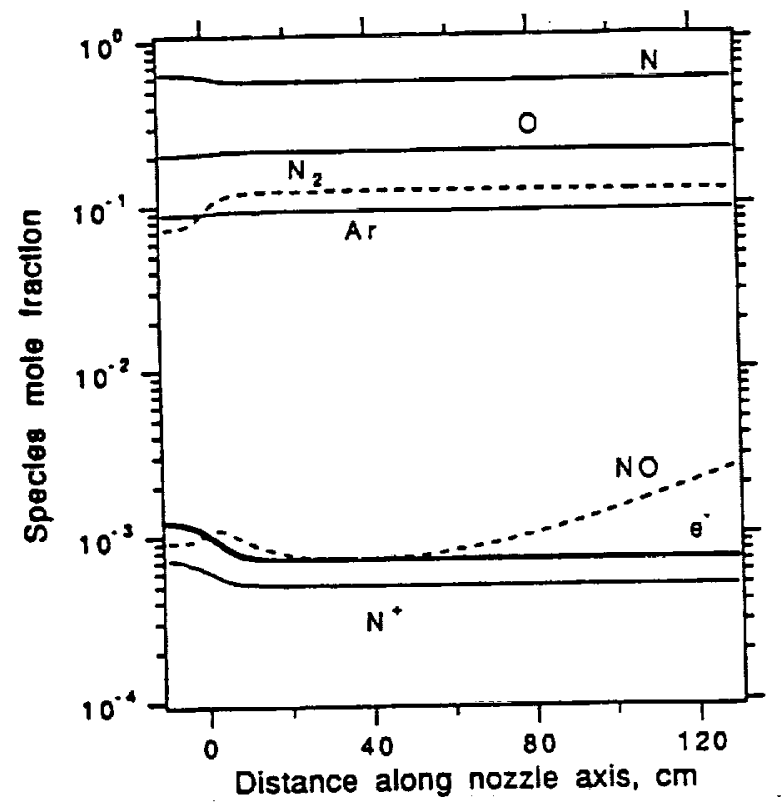

Fig. 7. (b) Species mole fractions.

The calculated equilibrium conditions in the shock layer are presented in Table 4 . The nonequilibrium processes in the blunt body shock layer are calculated using both one and two-dimensional codes. The one dimensional fow solution is obtained by the flow solver portion of the NONEQ code, which is the same as that identi- fied as STRAP in Ref. 7. The two-dimensional solution is obtained using a code referred to as 2D2T.F described in Ref. 16. Both codes use a two-temperature model in which the vibrational temperatures of the molecules, the electronic excitation temperatures of all species, and the electron thermal temperature are assumed to be the same. The one-dimensional code has been validated against experimental data in Ref. 7. The two-dimensional code has been validated indirectly by comparing with the onedimensional code. Both these codes were originally written for a freestream flow of undisturbed air. They were modified to accommodate the nonequilibrium freestream conditions of the present flow environment.

Table 4. Calculated equilibrium conditions in the shock layer.

\begin{tabular}{ll}
\hline Pressure & $0.0118 \mathrm{~atm}$ \\
Temperature & $6430 \mathrm{~K}$ \\
$\mathrm{Ar}$ mole fraction & $8.56^{-2}$ \\
$\mathrm{~N}_{2}$ mole fraction & $3.44^{-2}$ \\
$\mathrm{O}_{2}$ mole fraction & 0 \\
$\mathrm{NO}$ mole fraction & $1.34^{-4}$ \\
$\mathrm{~N}$ mole fraction & $6.79^{-1}$ \\
$\mathrm{O}$ mole fraction & $2.00^{-1}$ \\
$\mathrm{Ar}^{+}$mole fraction & $3.46^{-5}$ \\
$\mathrm{~N}^{+}$mole fraction & $5.07^{-4}$ \\
$\mathrm{O}^{+}$mole fraction & $1.59^{-4}$ \\
$\mathrm{NO}^{+}$mole fraction & $1.09^{-4}$ \\
$\mathrm{e}^{-}$mole fraction & $8.10^{-4}$ \\
\hline
\end{tabular}

The two codes were run for the $30 \mathrm{MJ} / \mathrm{kg}$ case and at higher enthalpies. The vibrational temperature of $\mathrm{N}_{2}$ determined by NOZNT code was chosen as representative of the vibrational-electron-electronic temperature ahead of the shock wave in the one- and the two-dimensional blunt body flow codes. A one-dimensional solution obtained for a constant-area channel is shown in Fig. 1, along with the total radiation emission power calculated using the NONEQ code.

The solution obtained from the one-dimensional code is shown in Fig. 8(a) and (b) for the $30 \mathrm{MJ} / \mathrm{kg}$ case. As seen in the figure, there is substantial variation in the temperatures and concentrations of the species across the shock layer. The two-dimensional code yielded approximately the same results.

\section{Spectral Calculation}

The spectral intensities are calculated for the flow properties shown in Figs. 8(a) and (b) using the spectral part of the NONEQ code. Presently the code allows calculation of nonequilibrium radiation only for nitrogen, oxygen, and carbon. Therefore presence of argon was neglected in the code. Figure 9 shows the comparison between the measured and the calculated spectra. As seen here, the calculation approximately reproduces the intensities of the atomic lines, but underestimates the background 
radiation composed of bound-free and free-free continua and molecular radiation.

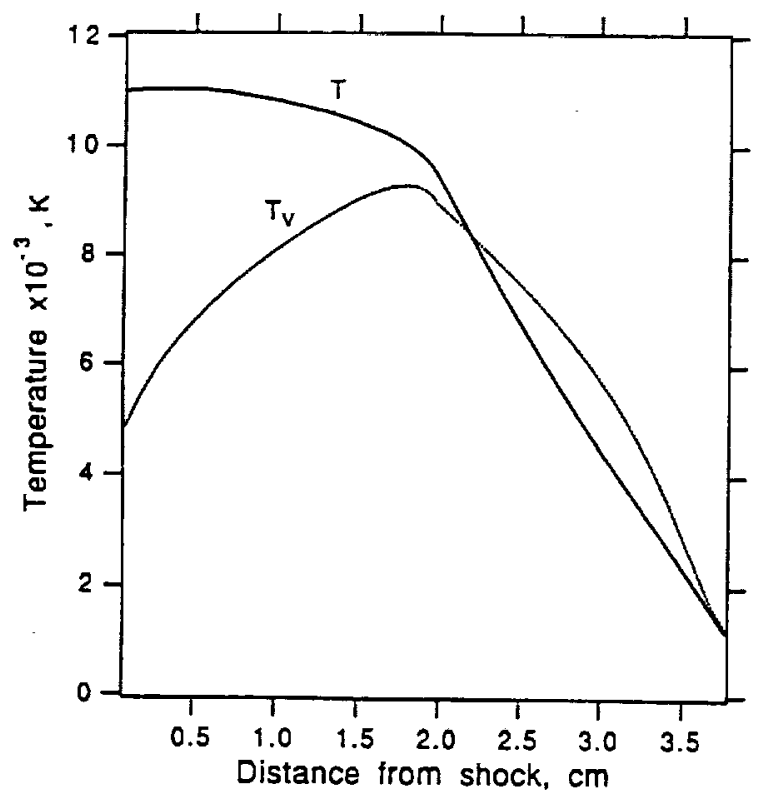

Fig. 8. Calculated properties along the stagnation line. (a) Temperatures.

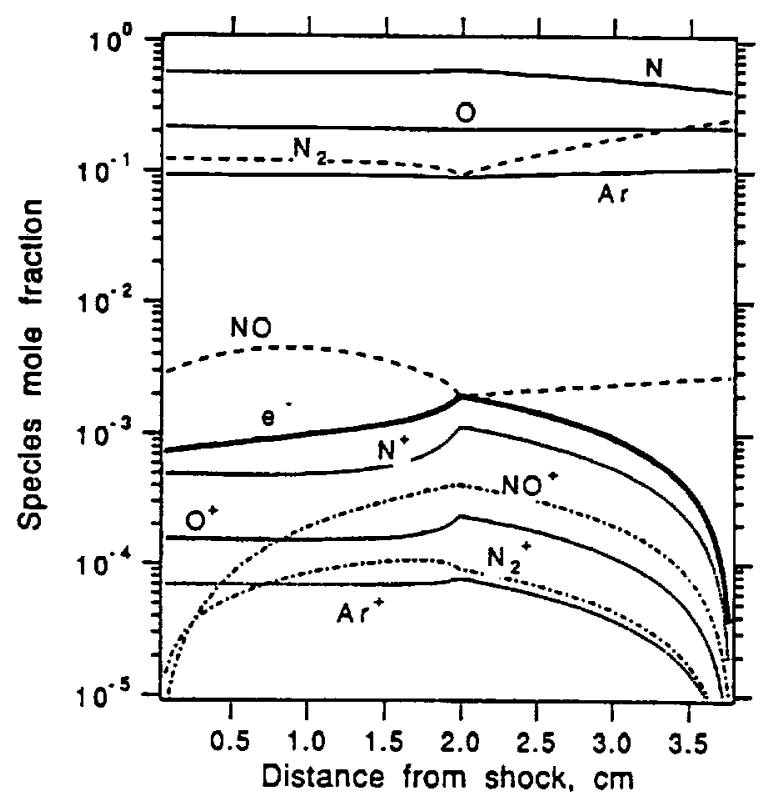

Fig. 8. (a) Species mole fractions.

In order to verify that this large discrepancy is not due to the misjudgment of the enthalpy values, calculations were made also with enthalpy values of 35,40 and 45 $\mathrm{MJ} / \mathrm{kg}$. At these higher enthalpies, the atomic line intensities are calculated to be higher, by a factor within about 3 , but the background radiation remained relatively unchanged. This behavior is explained as follows: at higher flow enthalpy, the electron concentration in the nozzle increases. This causes the electron temperature to approach the lower $\mathrm{N}_{2}$ vibrational temperature. Thus the initially low vibrational-electron-electronic temperature produces a slow rate of ionization behind the shock wave, which in turn produces only a small increase in electron density and continuum radiation behind the shock wave. Therefore, the low background radiation at higher enthalpy is not unreasonable.

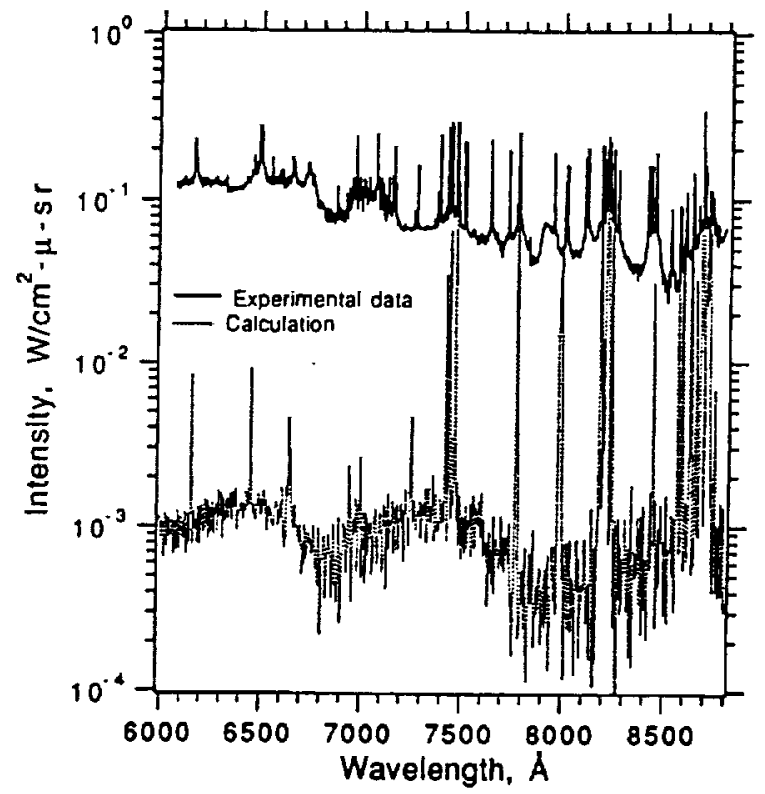

Fig. 9. Comparison between the measured and the calculated spectra.

\section{Discussion}

\section{Equivalent Intensities}

In order to explain the discrepancy between the experimental and theoretical spectral intensities, it is hypothesized that the ionization phenomena behind the bow shock wave occurs at a rate faster than calculated due to the highly excited freestream. Highly excited species require little energy to vibrationaly excite or ionize. Computationally, this situation is implemented by (i) making the vibrational relaxation times very short, (ii) lowering the magnitude of the energy feedback in electron-impact ionization (the energy depletion in the electron gas as a result of electron-impact ionization), and (iii) increasing the rate coefficients for electron-impact jonization reactions. The vibrational relaxation times are taken to be those corresponding to the elastic collisions; the energy feedback is reduced arbitrarily to $1 \%$ of the ionization potential; and the ionization rates are increased one thousand-fold.

In Fig. 10, the solution for the enhanced reaction rates is compared with the original solution. As seen here, there is a significant reduction in electron temperature in the peak region and an increase in total radiated power. Nevertheless, the changes were unable to bring the calculated values of the background radiation into agreement with 
the experimental values. In order to quantify the discrepancy between the calculated and the experimental values, the calculation was repeated with the intensities of each radiation sources multiplied by an arbitrary factor.

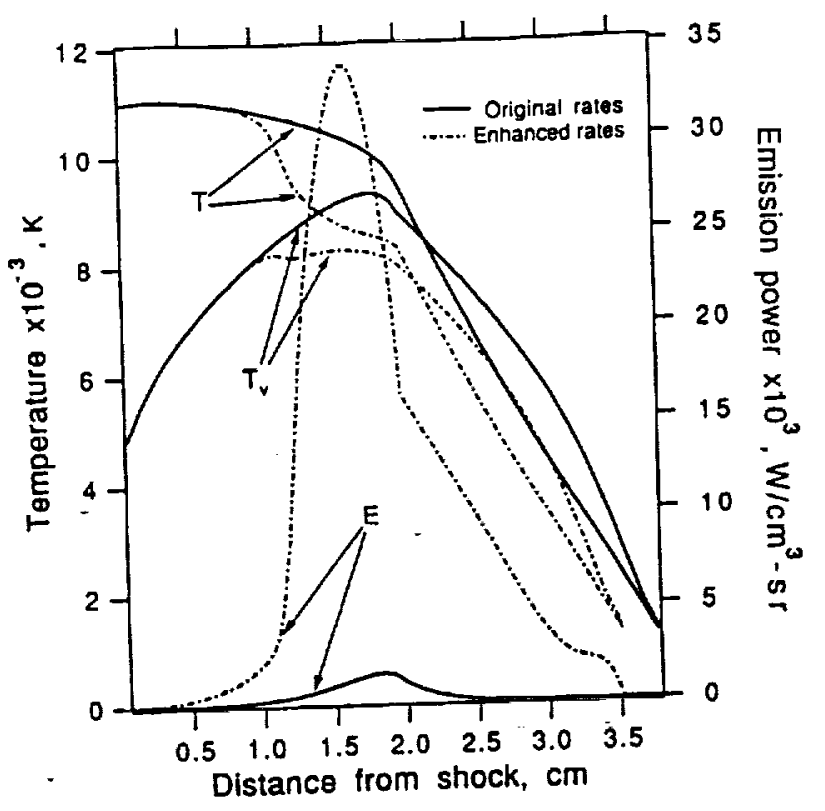

Fig. 10. Comparison between the solution for the enhanced reactions case and the original solution.

Through a trial and error procedure, the factors that gave the best agreement with the experimental data are given in Table 5 . The resulting synthetic spectrum is compared with the experimental data in Fig. 11.

Table 5. The multiplicative factors to reproduce the experimental spectrum.

\begin{tabular}{lr}
\hline Source & Factor \\
\hline $\mathrm{N}$ lines & 1 \\
$\mathrm{O}$ lines & 1 \\
$\mathrm{~N}^{+}+\mathrm{e}^{-}$bound-free continuum & 6000 \\
$\mathrm{O}^{+}+\mathrm{e}^{-}$bound-free continuum & 6000 \\
$\mathrm{~N}^{+}+\mathrm{e}^{-}$free-free continuum & 300 \\
$\mathrm{O}^{+}+\mathrm{e}^{-}$free-free continuum & 300 \\
$\mathrm{~N}_{2}$ First Positive band system & 50 \\
$\mathrm{~N}_{2}^{+}$First Negative band system & 500 \\
\hline
\end{tabular}

\section{Explanation of Discrepancy}

As shown in Fig. 1, the present experiment was conducted in a highly nonequilibrium regime where the available flow residence time is too short for the nonequilibrium radiation intensity to reach its peak point. As mentioned in Introduction, the flight experiments, Bow Shock 1 and Bow Shock 2, have demonstrated that calculations tend to severely underestimate radiation intensities in such a regime. In Fig. 12, the present results are compared with those of the two flight experiments. In the figure, the ratios of the calculated to the measured intensities, the reciprocals of the multiplicative factors in Table 2 , are shown as a function of flight altitude. Even though the equivalent flight altitude of the present test is $71.6 \mathrm{~km}$, it is shown as $61.2 \mathrm{~km}$. This is because the shock layer thickness in the present experiment is approximately four times that of the flight experiments, and therefore the equivalent freestream density is four times the experimental value for the same shock layer thickness.

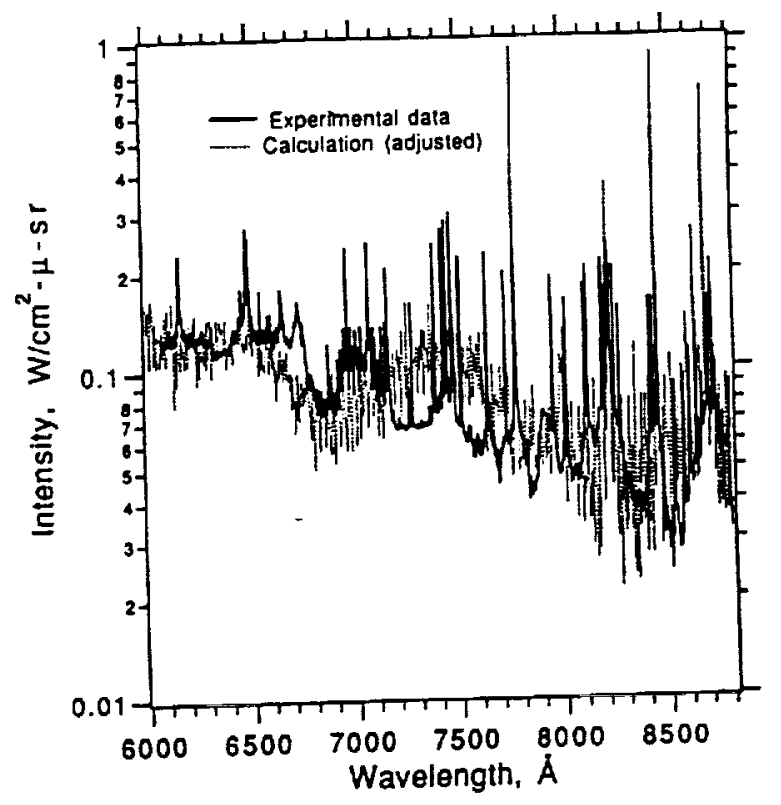

Fig. 11. Comparison between the measured spectra and the spectra calculated with enhanced reaction rates and intensity factors.

As indicated in Fig. 12, the calculated intensities are orders of magnitude smaller than the measured values in both the flight and the present experiments. The discrepancy between the theory and measurement on the intensity of the background radiation in the present experiment is comparable with that found for the NO Gamma band in the flight experiments. A discrepancy of similar extent is found in a shock tube experiment of Ref. 10 between the measured and the calculated intensities of the background radiation at wavelengths above $600 \mathrm{~nm}$.

The large discrepancy between the measurement and the calculation in the intensities of the bound-free and free-free continua might be due to the pseudo-continuum caused by the large numbers of weak atomic lines which are neglected in the calculation. The NONEQ code accounts for atomic lines listed in the NBS Tables. ${ }^{22}$ The Tables list only the lines from the states at or below the principal quantum number of 3 , and a few of those from the principal quantum number of 4 and 5 . However, there are a large number of atomic lines that emanate from the principal quantum number of 4 and all those from the principal quantum number above 4 that are unaccounted 
for in the NBS Tables. These neglected lines are mostly in the infrared wavelength region longer than $700 \mathrm{~nm}$. The widths of these lines are so broad that they appear as a continuum. Whether this pseudo-continuum can account for the missing radiation needs to be evaluated.

The discrepancy in the calculated intensities of the $\mathrm{N}_{2}$ First Positive and $\mathrm{N}_{2}^{+}$First Negative band systems is thought to be caused by the inadequacy of the code of the excitation code. The excitation portion of the NONEQ presently includes only four excited electronic states. The rate coefficients for collisional transitions among the states considered have been obtained mostly by extrapolation from the limited existing experimental data. Errors in the rate coefficients so determined, and the fact that there are transitions from many other electronic states, are believed to be responsible for this discrepancy. Such shortcomings of the NONEQ code can be corrected but it will require considerable additional work.

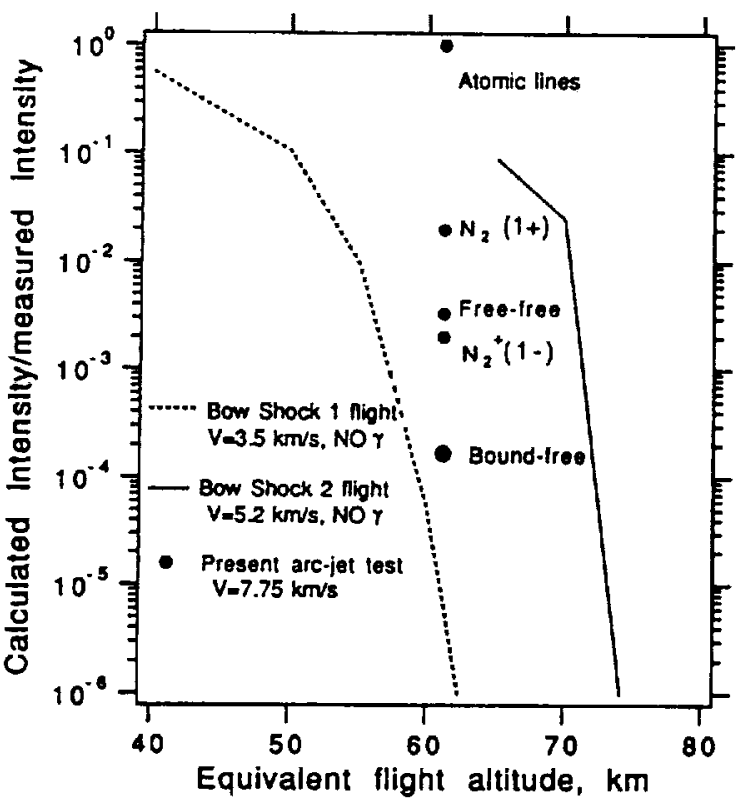

Fig. 12. The ratio between the calculated and the measured intensities of the present experiment compared with those for the Bow Shock 1 and Bow Shock 2 flight experiments.

The present work indicates that the features of the nonequilibrium spectrum seen in an arc-jet wind tunnel test are the same as those in flight or in a shock tube experiment. This is an indication that an arc-jet experiment can be used for the purpose of studying nonequilibrium radiation phenomena. Work is presently in progress to reduce the visible and the vacuum ultraviolet spectral data obtained in the experiments mentioned earlier.

\section{Conclusions}

The spectra of the shock layer radiation incident on the stagnation point of a blunt body were measured in an arcjet wind tunnel in the wavelength range from 600 to 880 $\mathrm{nm}$ in a highly nonequilibrium regime using an air-argon mixture as the test gas. Synthetic spectra were produced through the computation of the constricted-arc characteristics, nozzle flow, blunt body flow, and nonequilibrium radiation phenomena. Most of the calculated intensities of atomic lines agree approximately with the measured values, but those for continua and molecular bands are much weaker than the measured values. The discrepancy between the measured and the calculated intensities is similar to that found in two flight experiments and in shock tube data. Possible explanations for the discrepancy are presented. The present work shows also that an arc-jet wind tunnel can be used for the study of nonequilibrium radiation phenomenon.

\section{Acknowledgement}

This work was supported partially by a NASA Ames Director's Discretionary fund. The support for D.S.B. by NASA Grant NCC2-420, for G.P. by Grant NCC2653 , and for R.A.C. by Grant NCC2-762 are gratefully acknowledged.

\section{References}

${ }^{1}$ Park, C., "Radiation Enhancement by Nonequilibrium in Earth's Atmosphere," Journal of Spacecraft and Rockets, Vol. 22, No. 1, January-February 1985, pp. $27-$ 36.

${ }^{2}$ Park, C., Howe, J.T., Jaffe, R.L., and Candler, G. V., "Chemical Kinetic Problems of Future NASA Missions. 2. Mars Entries: A Review," To be published in Journal of Thermophysics and Beat Transfer.

${ }^{3}$ Davy, W., Park, C., Arnold, J. A., and Balakrishnan, A., "Radiometer Experiment For the Aeroassist Flight Experiment," AIAA Paper 85-0967, June 1985.

${ }^{4}$ Craig, R. A., Davy, W. C., and Whiting, E.E., "Science Objectives and Performance of a Radiometer and Window Design for Atmospheric Entry Experiments," to be published as a NASA TMX, 1994.

${ }^{3}$ Park, C., "Calculation of Nonequilibrium Radiation in the Flight Regimes of Aeroassisted Orbital Transfer Vehicles," Thermal Design of Aeroassisted Orbital Transfer Vehicles, Progress in Astronautics and Aeronautics, Vol. 96, edited by H. F. Nelson, AIAA, 1985, pp. 395-418.

${ }^{6}$ Park, C., "Nonequilibrium Air Radiation (NEQAIR) Program: User's Manual," NASA TM 86707, July 1985.

${ }^{7}$ Park, C., "Assessment of Two-Temperature Kinetic Model for lonizing Air," Journal of Thermophysics and Heat Transfer, Vol. 3, No. 3, July 1989, pp. 233-244.

${ }^{8}$ Whiting, E. E., and Park, C., "Radiative Heating at the Stagnation Point of the AFE Vehicle," NASA TM 102829, November 1990.

${ }^{9}$ Sharma, S. P., and Gillespie, W., "Nonequilibrium and Equilibrium Shock Front Radiation Measurements," Journal of Thermophysics and Heat Transfer, Vol. 5, No. 3, July-September 1991, pp. 257-265. 
${ }^{10}$ Sharma, S. P., Gillespie, W. D., and Meyer, S. A., "Shock Front Radiation Measurements in Air," AIAA Paper 91-0573, January 1991.

"Sharma, S. P., and Whiting, E. E., "Modelling of Nonequilibrium Radiation Phenomena: An Assessment," AIAA Paper 94-0253, January 1994.

${ }^{12}$ Sharma, S. P., "Assessment of Nonequilibrium Radiation Computation Method for Hypersonic Flows," International Journal of Modern Physics, Vol. 4, No. 4, April 1993, pp. 847-881.

${ }^{13}$ Levin, D. A., Candler, G. V., Collins, R. J., Erdman, P. W., Ziph, E. C., and Howlett, C. L., "Examination of Ultraviolet Radiation Theory for Bow Shock Rocket Experiments," AIAA Paper 92-2871, June 1992.

${ }_{14}$ Park, C., and Lee, S. H., "Validation of MultiTemperature Nonequilibrium Nozzle Flow Code NOZNT," AIAA Paper 93-2862, July 1993.

${ }_{15}$ Nicolet, W. E., Shepard, C. E., Clark, K. C., Balakrishnan, A., Kesselring, J. P., Suchsland, K.E., and Reese, J. J., "Methods for the Analysis of High-Pressure, High Enthalpy Are Beaters," AIAA Paper 75-0704, June 1975.

${ }^{16}$ Palmer, G., "Explicit Thermochemical Nonequilibrium Algorithm Applied to Compute Three-Dimensional
Aeroassist Flight Experiment Flowfields," Journal of Spacecraft and Rockets, Vol. 27, No. 5, SeptemberOctober, 1990.

${ }^{17}$ Palumbo, G., "Shock Layer Vacuum Ultraviolet Spectroscopy in an Arc-Jet Wind Tunnel," NASA TM 02258, January 1990.

${ }^{18}$ Palumbo, G., Craig, R., and Carrasco, A., "Spectral Measurements of Shock layer Radiation in an ArcJet Wind Tunnel," Paper 93-145, Instrument Society of America Meeting, Albuquerque, NM, April 1993.

${ }^{19}$ Craig, R. C., Palumbo, G., and Carrasco, A., "VUV Shock Layer Radiation in an Arc-Jet Wind Tunnel Experiment," NASA TM 108796, 1994.

${ }^{20}$ Durgapal, P., and Palmer, G., "Strongly Coupled Radiative Transfer and Joule Heating in the Cathode Region of an Arc Heater," AIA A Paper 93-2801, July 1993.

${ }^{21}$ Babikian D. S., Gopaul N. K. J. M., and Park, C., "Measurement and Analysis of Nitric Oxide Radiation in an Arc-Jet Flow," AIAA Paper 93-2800, July 1993.

${ }^{22}$ Wiese, W. L., Smith, M. W., and Glennon, B. M., "Atomic Transition Probabilities. Vol. 1. Hydrogen Through Neon," NSRDS-NBS 4, National Bureau of Standards, May 1966. 
APPENDIX - E 
-

-

$=$

픈 